\title{
Two-channel Kondo physics in two-impurity Kondo models
}

\author{
Andrew K. Mitchell, ${ }^{1}$ Eran Sela, ${ }^{1}$ and David E. Logan ${ }^{2}$ \\ ${ }^{1}$ Institute for Theoretical Physics, University of Cologne, 50937 Cologne, Germany \\ ${ }^{2}$ Department of Chemistry, Physical and Theoretical Chemistry, \\ Oxford University, South Parks Road, Oxford OX1 3QZ, United Kingdom
}

\begin{abstract}
We consider the non-Fermi liquid quantum critical state of the spin- $S$ two-impurity Kondo model, and its potential realization in a quantum dot device. Using conformal field theory (CFT) and the numerical renormalization group (NRG), we show the critical point to be identical to that of the two-channel Kondo model with additional potential scattering, for any spin- $S$. Distinct conductance signatures are shown to arise as a function of device asymmetry; with the 'smoking gun' square-root behavior, commonly believed to arise at low-energies, dominant only in certain regimes.

PACS numbers: 71.10.Hf, 73.21.La, 73.63.Kv
\end{abstract}

Systems comprising several quantum impurities inherently display an interplay between impurity-bath and interimpurity couplings [1 $[$ ]): the tendency for impurities to be Kondo-screened by conduction electrons competes with screening by interimpurity spin-singlet formation. Rich physics can thereby arise, as demonstrated for example in coupled quantum dots [7, 8], magnetic impurities in metals [1], and recent two-impurity STM experiments [9]. Indeed, the same essential physics governs the analogous propensities for heavy Fermion behavior or magnetic ordering in lattice systems [10].

The two-impurity, spin- $\frac{1}{2}$ Kondo model (2IKM) is the simplest to capture this competition [1]: local singlet formation is favored by an interimpurity exchange $K$, while coupling of each impurity to its own conduction channel favors separate Kondo screening below an effective singlechannel, single-impurity scale $T_{K}$ 2 [5]. The lack of interchannel charge transfer in the 2IKM permits two distinct phases, and a quantum phase transition (QPT) results on tuning $K_{c} \sim T_{K}$. At the critical point, non-Fermi liquid (NFL) physics arises below $T_{c}$, characterized [3] by anomalous properties such as fractional residual entropy and singular magnetic susceptibility.

This critical physics is also surprisingly robust to some perturbations, notably breaking of mirror (parity) symmetry or particle-hole symmetry [6]. Such perturbations are marginally irrelevant in the sense that the interimpurity coupling can be retuned to recover the critical point in all cases. But despite considerable effort, 2IKM critical physics has proved experimentally elusive - mainly due to interchannel charge transfer which smooths the QPT into a crossover [4]. Regular Fermi liquid (FL) physics then sets in below an energy scale $T^{*}$; and if the degree of charge transfer is large enough that $T^{*} \gg T_{c}$, no evidence of the critical point will be observed [11]. This is the situation relevant to the recent two-impurity experiments of Ref. [9]: coupling between one impurity on a metal surface and one on an STM tip was also accompanied by strong tip-surface tunneling.

Reducing the degree of interchannel charge transfer might be possible in a quantum dot device such as that proposed in Ref. [6]. Provided $T^{*} \ll T_{c}$, NFL behavior should be observable in an intermediate energy window, as can be understood from a 2IKM critical perspective (indeed the eventual crossover to FL physics is wholly characteristic of the intermediate NFL state [12]).

An alternative route could however involve use of a quantum box, which acts as an interacting lead [13]. Coupling a single dot to one regular lead and one box tuned to the Coulomb blockade regime, suppresses interchannel charge transfer completely. This has been exploited to access single-impurity two-channel Kondo (2CK) physics [14, 15] in a real device [13]. Here we propose simply to interject a second dot in series between the 'leads' to realize 2IKM physics. While parity and particle-hole symmetries are thereby broken, the QPT itself is unaffected. Robust NFL behavior should persist down to the lowest energy scales at the critical point.

Here we address two key questions in regard to potential realization of 2IKM physics. First, what is the nature of the critical point itself? We show that it is identical to that arising in a $2 \mathrm{CK}$ model with additional potential scattering, independent of parity breaking. Further, we show that the same QPT and 2CK critical point arises in the spin- $S$ generalization of the 2IKM. Second, what are the signatures of criticality in measurable quantities such as conductance? These reflect RG flow from higherenergy fixed points (FPs), and depend sensitively on parity breaking. We find in particular that the square-root behavior commonly anticipated [6, 7] at low-energies, is absent in the standard channel-symmetric 2IKM.

Nature of 2IKM critical point.- The 2IKM reads:

$$
H_{2 I K}=H_{0}+H_{p s}+J_{L} \vec{S}_{L} \cdot \vec{s}_{0 L}+J_{R} \vec{S}_{R} \cdot \vec{s}_{0 R}+K \vec{S}_{L} \cdot \vec{S}_{R},
$$

where $H_{0}=\sum_{\alpha, k} \epsilon_{k} \psi_{k \sigma \alpha}^{\dagger} \psi_{k \sigma \alpha}$ describes two free conduction channels $\alpha=L / R$, with density of states $\rho$, and spin density at the impurities $\vec{s}_{0 \alpha}=\sum_{\sigma \sigma^{\prime}} \psi_{0 \sigma \alpha}^{\dagger}\left(\frac{1}{2} \vec{\sigma}_{\sigma \sigma^{\prime}}\right) \psi_{0 \sigma^{\prime} \alpha}$ (where $\psi_{0 \sigma \alpha}^{\dagger}=\sum_{k} \psi_{k \sigma \alpha}^{\dagger}$ ). Potential scattering is included via $H_{p s}=\sum_{\alpha} V_{\alpha} \psi_{0 \sigma \alpha}^{\dagger} \psi_{0 \sigma \alpha}$, and $\vec{S}_{\alpha}$ are spin- $\frac{1}{2}$ operators for the impurities. The 2IKM has been extensively studied using a number of powerful techniques [2- 


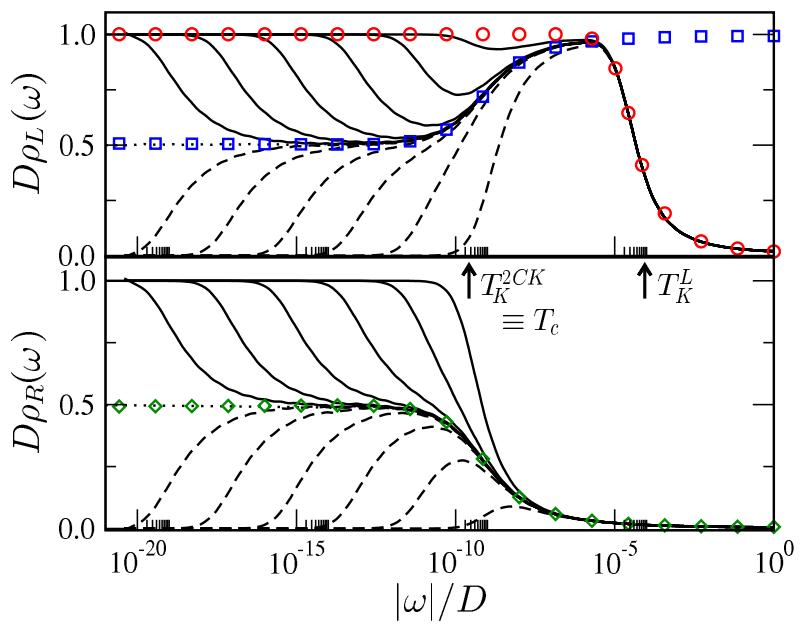

Figure 1: Spectra $D \rho_{\alpha}(\omega)$ vs frequency $|\omega| / D$ for channels $\alpha=L$ and $R$ [upper and lower panels] for the asymmetric $2 \mathrm{IKM}$ with fixed Kondo exchanges $\rho J_{L}=0.1, \rho J_{R}=0.05$, varying interimpurity exchange $K$ (and $H_{p s}=0$ ). Plotted using $K=K_{c}\left(1 \pm 10^{-n}\right)$ for dashed and solid lines, with integer $n=1 \rightarrow 6$ approaching progressively the critical point $K_{c} \sim T_{K}^{L} \approx 10^{-6} D$ (dotted line). Circles: for a $1 \mathrm{CK}$ model with $\rho J=\rho J_{L}$. Diamonds: $D \rho_{2 C K}(\omega)$ for a pure $2 \mathrm{CK}$ model with $T_{K}^{2 C K}=T_{c}$. Squares: $D \tilde{\rho}_{2 C K}(\omega)=1-D \rho_{2 C K}(\omega)$.

5, 12], and certain similarities have been found [46, 11, 12] between it and the 2CK model [14, 15],

$$
H_{2 C K}=H_{0}+H_{p s}+J_{L} \vec{S} \cdot \vec{s}_{0 L}+J_{R} \vec{S} \cdot \vec{s}_{0 R}
$$

with $\vec{S}$ a spin- $\frac{1}{2}$ operator for a single impurity, exchangecoupled to two independent conduction channels. The physics of the $2 \mathrm{CK}$ model is itself immensely rich [15]: the impurity is fully Kondo screened by the more stronglycoupled conduction channel below an effective singlechannel scale $T_{K}$, producing two distinct phases as a function of $\left(J_{L}-J_{R}\right)$. But when $J_{L}=J_{R}$, the frustration inherent when two channels compete to screen the impurity results in 'overscreening', and NFL physics results below $T_{K}^{2 C K}[15]$. Strikingly, both $2 \mathrm{CK}$ and 2IKM have the same fractional residual entropy, $S_{\mathrm{imp}}=\frac{1}{2} \ln (2)$.

CFT has been used to describe the critical points of both models [4, 16]. In the 'unfolded' representation, $H_{0}$ is written in terms of left-moving chiral Dirac Fermions. The CFTs for each model can be separated into different symmetry sectors. In particular, the $S U(2)_{2} \times$ $S U(2)_{2} \times U(1)$ flavor, spin and charge symmetries of the $2 \mathrm{CK}$ model [16], and the $U(1) \times U(1) \times S U(2)_{2} \times Z_{2}$ left/right charge, total spin and Ising symmetries of the 2IKM [3] can be exploited. The 2CK and 2IKM critical fixed point Hamiltonians take the same form as $H_{0}$, but with modified boundary conditions (BCs) that affect only the spin sector of the $2 \mathrm{CK}$ model [16] or the Ising sector of the 2IKM [4]. The finite size spectrum (FSS) at the critical point of each model, can then be deter- mined [4, 16]. In the channel-symmetric case $J_{L}=J_{R}$ and for $H_{p s}=0$, the FSS of the $2 \mathrm{CK}$ critical point is characterized by the fractions $0, \frac{1}{8}, \frac{1}{2}, \frac{5}{8}, 1 \ldots$; while for 2IKM a different FSS arises: $0, \frac{3}{8}, \frac{1}{2}, \frac{7}{8}, 1 \ldots$

Despite these apparent differences between the critical FPs of the two models, the 2IKM can be mapped onto an effective $2 \mathrm{CK}$ model in special cases [5, 6, 11]. The key requirement for that mapping is of course the generation of an effective spin- $\frac{1}{2}$ local moment (LM), which can then be overscreened by symmetric coupling to two conduction channels. In the channel-asymmetric limit $J_{L} \gg J_{R}, 2 \mathrm{CK}$ critical physics arises via a simple mechanism [6], first involving Kondo screening of the $L$ impurity by the $L$-lead on the single-channel scale $T_{K}^{L}$; followed by second-stage overscreening of the $R$-impurity by the $R$-lead and an effective coupling to the remaining Fermi liquid bath states of the $L$-lead below $T_{c}$. An effective 2CK model of form Eq. 2, valid at low-energies $T \lesssim T_{K}^{L}$, can be derived formally using the approach of Ref. [17], exploiting the Wilson chain representation [18]; and effective couplings follow as $\rho J_{L}^{\text {eff }} \sim K / T_{K}^{L}$ and $\rho J_{R}^{\text {eff }} \sim\left[1 / \rho J_{R}-1 / \rho J_{L}\right]^{-1}$. The $2 \mathrm{CK}$ FP is thus stable when $K=K_{c} \sim T_{K}^{L} \rho J_{R}^{e f f}$, so the low-energy physics of the $2 \mathrm{IKM}$ is in this case wholly equivalent to that of the $2 \mathrm{CK}$ model.

Importantly however, the $L$-channel free electrons in the effective $2 \mathrm{CK}$ model acquire a $\pi / 2$ phase shift due to the first-stage single-channel Kondo screening of the $L$-impurity in the original $2 \mathrm{IKM}$. This is seen clearly in the dynamics of the asymmetric 2IKM; to demonstrate which, and to highlight the basic physical picture, Fig. 1 shows spectra $D \rho_{\alpha}(\omega) \equiv-\pi \rho \operatorname{Im}\left[t_{\alpha}(\omega)\right]$ vs $|\omega| / D$ with $t_{\alpha}(\omega)$ the scattering t matrix [19]. Results are obtained from NRG, exploiting all model symmetries, discretizing conduction bands of width $2 D$ logarithmically using $\Lambda=$ 3 , and retaining 8000 states per iteration in each of $z=3$ interleaved calculations (for a review, see Ref. [18]).

Single-channel Kondo screening of the $L$-impurity by the $L$ lead on the scale of $T_{K}^{L}$ is seen directly in the $L$ spectra in the upper panel: a Kondo resonance, reaching the unitarity limit $D \rho_{L}=1$, which has precisely the form of a regular single-channel Kondo (1CK) model [10]. This embodies the $\pi / 2$ phase shift in the $L$ channel; but no such feature is observed on this energy scale in the $R$ spectra (lower panel), indicating that the $R$-impurity is still essentially free. On tuning the interimpurity coupling $K$ closer to the critical point of the 2IKM, the spectra in both channels fold progressively onto the critical spectra. For energies $|\omega| / D \ll T_{K}^{L}$, the critical spectrum $D \rho_{R}(\omega)$ is precisely that of a $2 \mathrm{CK}$ model $D \rho_{2 C K}(\omega)$ with $T_{K}^{2 C K}=T_{c}$. But to leading order the critical spectrum in the left channel is $D \rho_{L}(\omega)=1-D \rho_{2 C K}(\omega)$ [17]. Thus, at the channel-asymmetric critical point [6],

$$
D \rho_{\alpha}(\omega) \stackrel{|\omega| \ll T_{c}}{\sim} \frac{1}{2}+\alpha \beta \sqrt{|\omega| / T_{c}}
$$


with $\alpha= \pm 1$ for channel $L / R$ resulting from the additional $L$-channel $\pi / 2$ phase shift, and $\beta$ a constant $\mathcal{O}(1)$.

This phase shift can be included in the $2 \mathrm{CK}$ model, Eq. 2, via the potential scattering term $H_{p s}$ (ie $V_{L} \rightarrow \infty$ but $V_{R}=0$, accompanied also by retuning $J_{L}$ and $J_{R}$ to access the critical point). This is equivalent to adding infinite uniform and staggered potential scatterings, which affect respectively the charge and flavor sectors of the 2CK model. Modifying the CFT for the critical point of the $2 \mathrm{CK}$ model to include this, we find [20] the $\mathrm{BC}$ becomes equivalent to that of the 2IKM. The FSS is also naturally affected and is given by [20]

$$
E_{2 C K}=\frac{1}{8}(Q-a)^{2}+\frac{1}{4} j(j+1)+\frac{1}{4} j_{F}\left(j_{F}+1\right)-b j_{F}^{z},
$$

where $Q, j$ and $j_{F}$ are the charge, spin and flavor quantum numbers. Uniform potential scattering shifts the charge parabolas, while staggered potential scattering biases the flavor sector. The $\pi / 2$ phase shift in the $L$ channel corresponds to $a=1$ and $b=\frac{1}{2}$ [20]. Only certain quantum number combinations are allowed at the critical point, as given by the nontrivial gluing conditions derived in Ref. [16]; and which reproduce fully the 2IKM spectrum when used with Eq. 4 (see Fig. 2 of [20]). One remarkable result obtained from our NRG calculations [20] is that the FSS at the critical point of the 2IKM does not depend on channel asymmetry (whence in particular the critical point possesses an emergent parity symmetry, irrespective of bare model symmetries). Further, we have shown [20] that the critical point for one model with potential scattering $V_{L}$ and $V_{R}$ is equivalent to the critical point of the other model with different potential scattering $\tilde{V}_{L}$ and $\tilde{V}_{R}$. The $2 \mathrm{IKM}$ and $2 \mathrm{CK}$ critical FPs are thus equivalent in the sense that they lie on the same marginal NFL manifold parametrized by $H_{p s}$.

Conductance lineshapes and symmetry.- Full RG flow from the local moment (LM) FP to the 2CK FP is thus recovered at the critical point of the asymmetric 2IKM. This is manifest [6] in the conductance arising e.g. when a given channel $\alpha=L / R$ is split into source and drain. At zero-bias, it is given exactly 21] in terms of the scattering $t$ matrix (considered for the channel-asymmetric case in Fig. 1) by $G_{2 I K}^{\alpha}\left(V_{s d}=\right.$ $0, T) /\left(2 e^{2} h^{-1} G_{0}^{\alpha}\right)=-\int_{-\infty}^{\infty} d \omega \partial f(\omega / T) / \partial \omega D \rho_{\alpha}(\omega, T) ;$ with $f(\omega / T)$ the Fermi function, and the impurity-lead coupling parametrized by $G_{0}^{\alpha}=4 \Gamma_{s}^{\alpha} \Gamma_{d}^{\alpha} /\left(\Gamma_{s}^{\alpha}+\Gamma_{d}^{\alpha}\right)^{2}$, in terms of the $\alpha=L / R$ hybridizations to source $\left(\Gamma_{s}^{\alpha}\right)$ and drain $\left(\Gamma_{d}^{\alpha}\right)$. Indeed, in the limit $\Gamma_{s}^{\alpha} \gg \Gamma_{d}^{\alpha}$ (ie. $\left.G_{0}^{\alpha} \ll 1\right)$, the $T=0$ conductance follows as $\tilde{G}_{2 I K}^{\alpha}\left(V_{s d}\right)=$ $G_{2 I K}^{\alpha}\left(V_{s d}, T=0\right) /\left(2 e^{2} h^{-1} G_{0}^{\alpha}\right)=D \rho_{\alpha}\left(\omega=V_{s d}, T=0\right)$, and hence from Eq. 3 one finds at low energies $V_{s d} \ll T_{c}$,

$$
\tilde{G}_{2 I K}^{\alpha}\left(V_{s d}\right)=\frac{1}{2}+\alpha \beta \sqrt{V_{s d} / T_{c}}+\gamma_{\alpha}\left(V_{s d} / T_{c}\right)+\ldots
$$

where we include also a term linear in $V_{s d} / T_{c}$.

The leading square-root behavior of Eq. 5 has been viewed as the 'smoking gun' signature of this $2 \mathrm{CK}$

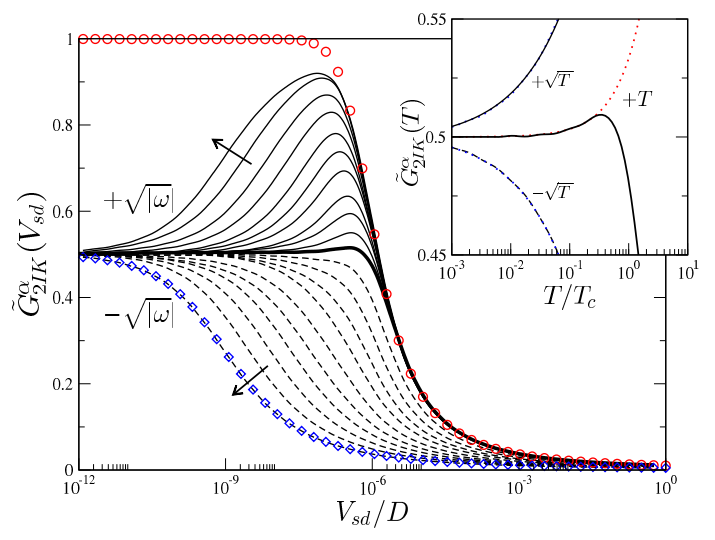

Figure 2: $T=0$ conductance $G_{2 I K}^{\alpha} /\left(2 e^{2} h^{-1} G_{0}^{\alpha}\right)$ through channel $\alpha=L$ and $R$ (solid and dashed lines) vs bias $V_{s d} / D$, at the critical point. Shown for $\rho J_{L}=0.075 \geq \rho J_{R}$, varying $\rho J_{R}=0.075 \rightarrow 0.05$ in steps of 0.0025 , with $K=K_{c} \sim T_{K L}^{1 C K}$ retuned in each case (and $H_{p s}=0$ ). Thick solid line is the symmetric case $J_{L}=J_{R}$; asymmetry $J_{L} / J_{R} \geq 1$ increases in direction of arrows. Circle and diamonds: pure $1 \mathrm{CK}$ and $2 \mathrm{CK}$ scaling spectra. Inset: zero-bias conductance vs $T / T_{c}$ for $J_{L} / J_{R}=1$ and 2 (and $\alpha=L, R$ ), exhibiting respectively leading linear and square-root behavior (dotted lines).

physics [6, 22], and was used to identify the critical point in the 2CK experiment of Ref. 13]. But we note that, unlike the $2 \mathrm{CK}$ model, the $2 \mathrm{IKM}$ does not possess $S U(2)$ flavor symmetry. Since symmetry dictates which operators can act in the vicinity of the critical FP, this is naturally reflected in the asymptotic conductance through the coefficients $\beta$ and $\gamma_{\alpha}$. Indeed, the full energy-dependence of conductance depends on the unstable FPs, whose vying effects on RG flow again depend on symmetry and model parameters. For example, in the usual symmetric 2IKM (Eq. 1 with $J_{L}=J_{R}$ and $H_{p s}=0$ ), no incipient LM is formed: there is no intermediate energy window with eg. $S_{\mathrm{imp}}=\ln (2)$ entropy, and RG flow proceeds directly to the $2 \mathrm{CK}$ FP from the $\mathrm{LM} \times \mathrm{LM}$ high energy FP describing a pair of free impurities $\left(S_{\mathrm{imp}}=\ln (4)\right)$.

The effect of parity-breaking is explored in Fig. 2, showing NRG results for conductance vs bias $V_{s d}$ at the 2IKM critical point (obtained for $G_{0}^{\alpha} \ll 1$, as above). Conductance in the asymmetric limit $J_{L} \gg J_{R}$ is consistent with Ref. [6], and physical expectation as above (see Eq. 5). Here, the asymptotic conductance is $\tilde{G}_{2 I K}^{R}\left(V_{s d}\right) \simeq 1-\tilde{G}_{2 I K}^{L}\left(V_{s d}\right) \equiv \tilde{G}_{2 C K}\left(V_{s d}\right)$ at low energies $V_{s d} \ll T_{K}^{L}$, where $T_{K}^{L} \gg T_{c} \equiv T_{K}^{2 C K}$ and with $\tilde{G}_{2 C K}\left(V_{s d}\right)$ the conductance of the standard 2CK model [13, 22] (diamonds). Thus, on exchanging $J_{R} \leftrightarrow J_{L}$, the coefficient $\beta$ of Eq. 5 must change sign. But what happens as the asymmetry is decreased? We find the leading square-root contribution in Eq. (5) vanishes (see Fig. 2), as $\beta \sim\left(J_{L}-J_{R}\right)$, and leading linear behavior emerges at the symmetric point $J_{L}=J_{R}$ (the same naturally arising as a function of $T$ at zero-bias, see Fig. 2 inset). In fact 


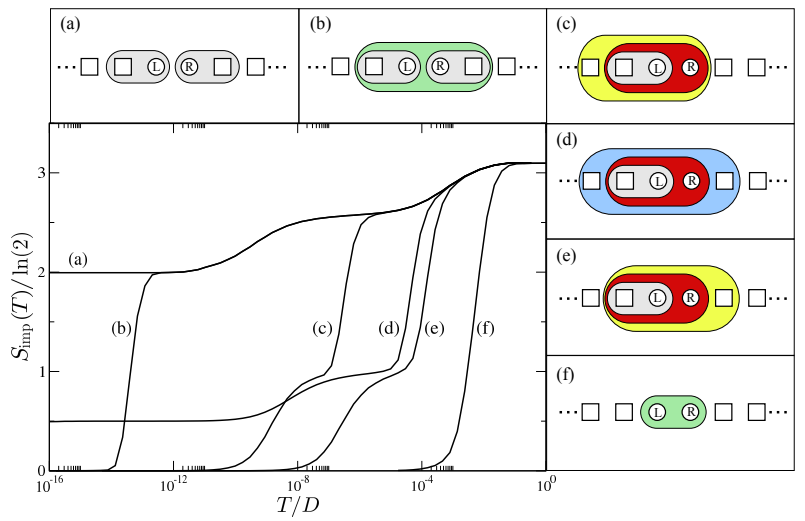

Figure 3: Impurity entropy $S_{\text {imp }}(T)$ vs $T / D$ for the 2 IKM with spin-1 impurities. Plotted for fixed $\rho J_{L}=0.15, \rho J_{R}=$ 0.05 , varying $K / D=0,10^{-13}, 3 \times 10^{-7}, K_{c} \approx 6 \times 10^{-5}, 2 \times$ $10^{-4}$ and $10^{-2}$ for lines (a)-(f). The corresponding physical processes are illustrated in panels (a)-(f): impurities denoted as circles and conduction band orbitals (in the Wilson chain representation [18]) as squares. For discussion, see text.

linear- $V_{s d}$ behavior also emerges as the symmetric point is approached, since the square-root term dominates over a shrinking window $V_{s d} / T_{c} \ll\left(\beta / \gamma_{\alpha}\right)^{2}$.

Another striking feature of the conductance in more channel-symmetric situations is the behavior at higher $T$ or energies $\gtrsim T_{c} \approx T_{K}^{L}$. Here the behavior is wholly characteristic of single-impurity, single-channel Kondo physics, as seen by comparison to the circles in Fig. 2 .

The absence of square-root behavior in conductance of the symmetric $2 \mathrm{IKM}$ is contrary to common belief [6, 7], so we sketch now our CFT proof [20]. As pointed out in Refs. [4, 6], corrections to the t matrix in the vicinity of the critical point (whose $\omega$-dependence displays the same scaling as conductance) are determined from irrelevant boundary operators consistent with symmetry. Two such play a role here: $\delta H_{1}=c_{1} \epsilon^{\prime}$ and $\delta H_{2}=c_{2} \vec{J}_{-1} \cdot \vec{\phi}$ (in the notation of Ref. [4]). The operator $\vec{J}_{-1} \cdot \vec{\phi}$ is the leading irrelevant operator of the $2 \mathrm{CK} \mathrm{FP}$, whose effect on the $\mathrm{t}$ matrix is known [23] to yield the famous square-root behavior. However, $\vec{J}_{-1} \cdot \vec{\phi}$ has odd parity in the $2 \mathrm{IKM}$ (unlike $2 \mathrm{CK}$ ), which implies that its coefficient $c_{2} \sim\left(J_{L}-\right.$ $\left.J_{R}\right)$ vanishes in the symmetric limit. In both models, $\delta H_{1}$ does still contribute ( $c_{1}$ always being finite [4]). One might naively expect $\epsilon^{\prime}$ to behave similarly to $\vec{J}_{-1} \cdot \vec{\phi}$ since they have the same scaling dimension $3 / 2$. However, the key difference between $\epsilon^{\prime}$ and $\vec{J}_{-1} \cdot \vec{\phi}$ is that only the latter is a Virasoro primary field. In consequence [20], the leading square-root correction to the t matrix from $\delta H_{1}$ vanishes. In the symmetric $2 \mathrm{IKM}$, the leading squareroot behavior of conductance thus also vanishes.

Spin-S 2IKM.- Multilevel quantum dots can behave like $S=1$ impurities [24], and high-spin impurities such as Co $(S=3 / 2)$ have been manipulated with STM [25].
Thus a natural and pertinent generalization of the 2IKM involves spin- $S$ impurities: the model remains Eq. 1, but $\vec{S}_{R}, \vec{S}_{L}$ are now spin- $S$ operators.

A QPT must again arise, as follows from the same line of argument as the spin- $\frac{1}{2}$ 2IKM [3]. On tuning $K$ there is a phase-shift discontinuity on going from the local singlet phase for large $K$ to a separated spin- $S$ underscreened Kondo phase for small $K$. The nature of the transition arising at $K_{c}$ is again clear by considering the asymmetric limit $J_{L} \gg J_{R}$. For concreteness consider $S=1$, although the argument extends easily to higher$S$. NRG results for the entropy $S_{\mathrm{imp}}(T)$ vs $T$ are shown in Fig. 3, together with cartoons highlighting the key physical processes. In (a) the impurities are completely decoupled $(K=0)$, with each thus underscreened to a spin- $\frac{1}{2}$ by its attached lead $\alpha$, on its own single-channel Kondo scale $T_{K}^{\alpha}$ (with residual entropy $2 \ln (2)$ ). For small finite $K<T_{K}^{R}$, (b), these residual moments form a local singlet state on the scale $T \sim K$, so the residual entropy is quenched. On increasing the interimpurity $K$ further $\left(T_{K}^{R}<K<T_{K}^{L}\right)$, the underscreened spin- $\frac{1}{2} L$ impurity and the still unscreened spin-1 $R$ impurity are coupled and form a local doublet state on the scale $T \sim K$. This can then be single-channel Kondo screened by an effective coupling either to the $L$ channel (c) or the $R$ channel (e), and the residual entropy is again quenched. However, $L$ and $R$ effective couplings can become equal on finetuning $K$. This is the single spin- $\frac{1}{2} 2 \mathrm{CK}$ critical point (d), with residual entropy $\frac{1}{2} \ln (2)$. For large $K \gg T_{K}^{L}$, a local interimpurity singlet state arises as expected (f).

Analysis of the finite size spectrum at the critical point shows it to be identical to that of the regular spin- $\frac{1}{2}$ 2IKM, independent of asymmetry [20]; and is hence that of a $2 \mathrm{CK}$ model with additional potential scattering.

Conclusion.- We have shown the critical point of the spin- $S$ IIKM, including the spin- $\frac{1}{2}$ variant, to be ubiquitously $2 \mathrm{CK}$ in nature. However conductance lineshapes measurable in experiment exhibit distinctive behavior depending on underlying symmetries, the low-energy behavior in particular evolving from square-root to linear behavior in $V_{s d}$ or $T$ as the channel symmetric point is approached, and for any spin- $S$.

Acknowledgments.- We thank I. Affleck and T. Quella for insightful input, and acknowledge financial support from the DFG through SFB608 and FOR960 (AKM), the A. v. Humboldt foundation (ES), and EPSRC through $\mathrm{EP} / \mathrm{I} 032487 / 1$ (DEL).

[1] C. Jayaprakash, H. R. Krishnamurthy, and J. W. Wilkins, Phys. Rev. Lett. 47, 737 (1981).

[2] B. A. Jones and C. M. Varma, Phys. Rev. Lett. 58, 843 (1987).

[3] I. Affleck and A. W. W. Ludwig, Phys. Rev. Lett. 68, 1046 (1992). 
[4] I. Affleck, A. W. W. Ludwig, and B. A. Jones, Phys. Rev. B 52, 9528 (1995).

[5] J. Gan, Phys. Rev. Lett. 74, 2583 (1995).

[6] G. Zaránd, C.-H. Chung, P. Simon, and M. Vojta, Phys. Rev. Lett. 97, 166802 (2006).

[7] A. M. Chang and J. C. Chen, Rep. Prog. Phys. 72, 096501 (2009).

[8] W. G. van der Wiel et al., Rev. Mod. Phys. 75, 1 (2003).

[9] J. Bork et al., Nature Physics 7, 901 (2011).

[10] A. C. Hewson, The Kondo Problem to Heavy Fermions (Cambridge University Press, Cambridge, 1993).

[11] F. W. Jayatilaka, M. R. Galpin, and D. E. Logan, Phys. Rev. B 84, 115111 (2011).

[12] E. Sela, A. K. Mitchell, and L. Fritz, Phys. Rev. Lett. 106, 147202 (2011).

[13] R. M. Potok, I. G. H. Shtrikman, Y. Oreg, and D. Goldhaber-Gordon, Nature (London) 446, 167 (2007).

[14] P. Nozières and A. Blandin, J. Phys. (Paris) 193, 41 (1980).

[15] D. L. Cox and A. Zawadowski, Adv. Phys. 47, 599 (1998).
[16] I. Affleck and A. W. W. Ludwig, Nucl. Phys. B 360, 641 (1990).

[17] A. K. Mitchell, D. E. Logan, and H. R. Krishnamurthy, Phys. Rev. B 84, 035119 (2011).

[18] R. Bulla, T. Costi, and T. Pruschke, Rev. Mod. Phys. 80, 395 (2008).

[19] A. K. Mitchell and D. E. Logan, Phys. Rev. B 81, 075126 (2010).

[20] See supplementary material.

[21] Y. Meir and N. S. Wingreen, Phys. Rev. Lett. 68, 2512 (1992).

[22] M. Pustilnik, L. Borda, L. I. Glazman, and J. von Delft, Phys. Rev. B 69, 115316 (2004).

[23] I. Affleck and A. W. W. Ludwig, Phys. Rev. B 48, 7297 (1993).

[24] D. E. Logan, C. J. Wright, and M. R. Galpin, Phys. Rev. B 80, 125117 (2009).

[25] A. F. Otte et al., Nature Physics 4, 847 (2008). 


\title{
Two-channel Kondo physics in two-impurity Kondo models: Supplementary Material
}

\author{
Andrew K. Mitchell, ${ }^{1}$ Eran Sela, ${ }^{1}$ and David E. Logan ${ }^{2}$ \\ ${ }^{1}$ Institute for Theoretical Physics, University of Cologne, 50937 Cologne, Germany \\ ${ }^{2}$ Department of Chemistry, Physical and Theoretical Chemistry, \\ Oxford University, South Parks Road, Oxford OX1 3QZ, United Kingdom
}

The following appendices comprise the supplementary material to Ref. 1.

\section{CONNECTING THE FINITE SIZE SPECTRA OF THE TWO-CHANNEL AND TWO-IMPURITY KONDO MODELS}

In this appendix we demonstrate that the critical points of the 2CK model and 2IKM are connected by a marginal operator corresponding to the potential scattering $H_{p s}$. Specifically, the finite size spectrum of one model is shown to transform into that of the other model when this additional term is accounted for explicitly. In the channel-asymmetric $2 \mathrm{IKM}$, the physical origin of this operator is readily understood from the mapping 2 between $2 \mathrm{IKM}$ and $2 \mathrm{CK}$, where an additional phase shift in the more strongly-coupled channel arises due to first-stage single-channel Kondo screening of one of the impurities.

First, we review how the finite size spectrum at the critical point of the $2 \mathrm{CK}$ model is organized according to the unperturbed CFT with $U(1) \times S U(2)_{2} \times S U(2)_{2}$ charge, spin and flavor symmetry sectors. The spectrum consists of Kac-Moody conformal towers, obtained by combining these symmetry sectors according to the gluing conditions ${ }^{3}$ shown in Table [. The energy of the lowest lying state in each conformal tower (measured in units of $\frac{2 \pi \hbar v_{F}}{L}$, with $v_{F} \equiv 1$ the Fermi velocity and $\hbar \equiv 1$ hereafter) follows from the formula ${ }^{3}$

$$
E_{2 C K}=\frac{Q^{2}}{8}+\frac{j(j+1)}{4}+\frac{j_{F}\left(j_{F}+1\right)}{4},
$$

where $Q$ is the total charge, $j=0, \frac{1}{2}, 1$ is the total spin, and $j_{F}=0, \frac{1}{2}, 1$ is the flavor quantum number. Only certain quantum number combinations are allowed at the $2 \mathrm{CK}$ critical point, $\stackrel{3}{=}$ as given by the nontrivial gluing conditions of Table【 (and with level degeneracies also following from the Table). Also note that the gluing conditions for integer $Q$ are defined modulo 2. Each conformal tower has an infinite number of states. Consider for example the spin $j$ tower of the spin $S U(2)_{2}$ sector. The states with lowest energy form a spin $j$ representation of $S U(2)$, and hence are $(2 j+1)$ degenerate (the corresponding magnetic quantum number is $j_{z}=-j,-j+1, \ldots, j$ ). The reducible representations of $S U(2)$ with increasing spin (equal to $j$ modulo 1 ) give rise to higher energy states. In the space of $j_{z}$ and energy $n$, the envelope of an $S U(2)_{k}$ conformal tower on level $k$ with spin $j$ is $n=\left[\left(j^{z}\right)^{2}-j^{2}\right] / k^{4}$. For a visualization of such unperturbed conformal towers, see the upper panels of Fig. 1]

We now perturb the CFT by introducing potential scattering $H_{p s}=\sum_{\alpha} V_{\alpha} \psi_{0 \sigma \alpha}^{\dagger} \psi_{0 \sigma \alpha}$. To see how this perturbation enters in the CFT, we first switch off the Kondo interactions, and consider free Fermions of a single species $\sigma \alpha$. With antiperiodic boundary conditions $\psi_{\sigma \alpha}(-L / 2)=-\psi_{\sigma \alpha}(L / 2)$, the single particle momenta are $k=\frac{2 \pi}{L}\left(n+\frac{1}{2}\right)$; and for linear dispersion, the single particle energies are just given by $k$. The potential scattering amplitudes $V_{\alpha}$ can be parametrized in terms of scattering phase shifts $\delta_{\alpha}$. They are defined in terms of the shift of these single particle levels $k=\frac{2 \pi}{L}\left(n+\frac{1}{2}-\frac{\delta_{\sigma \alpha}}{\pi}\right)$. For $N_{\sigma \alpha}$ particles of each species, the correction to the total energy (again in units of $\frac{2 \pi}{L}$ )

\begin{tabular}{|rrrrr|}
\hline$Q$ & $j$ & $j_{F}$ & multiplicity & $E_{2 C K}$ \\
\hline 0 & $1 / 2$ & 0 & 2 & $3 / 16$ \\
\pm 1 & 0 & $1 / 2$ & 4 & $5 / 16$ \\
\pm 1 & 1 & $1 / 2$ & 12 & $13 / 16$ \\
\pm 2 & $1 / 2$ & 0 & 4 & $11 / 16$ \\
0 & $1 / 2$ & 1 & 6 & $11 / 16$ \\
\hline
\end{tabular}

TABLE I: Nontrivial gluing conditions of conformal towers belonging to the $U(1) \times S U(2)_{2} \times S U(2)_{2}$ charge, spin and flavor symmetry sectors. 

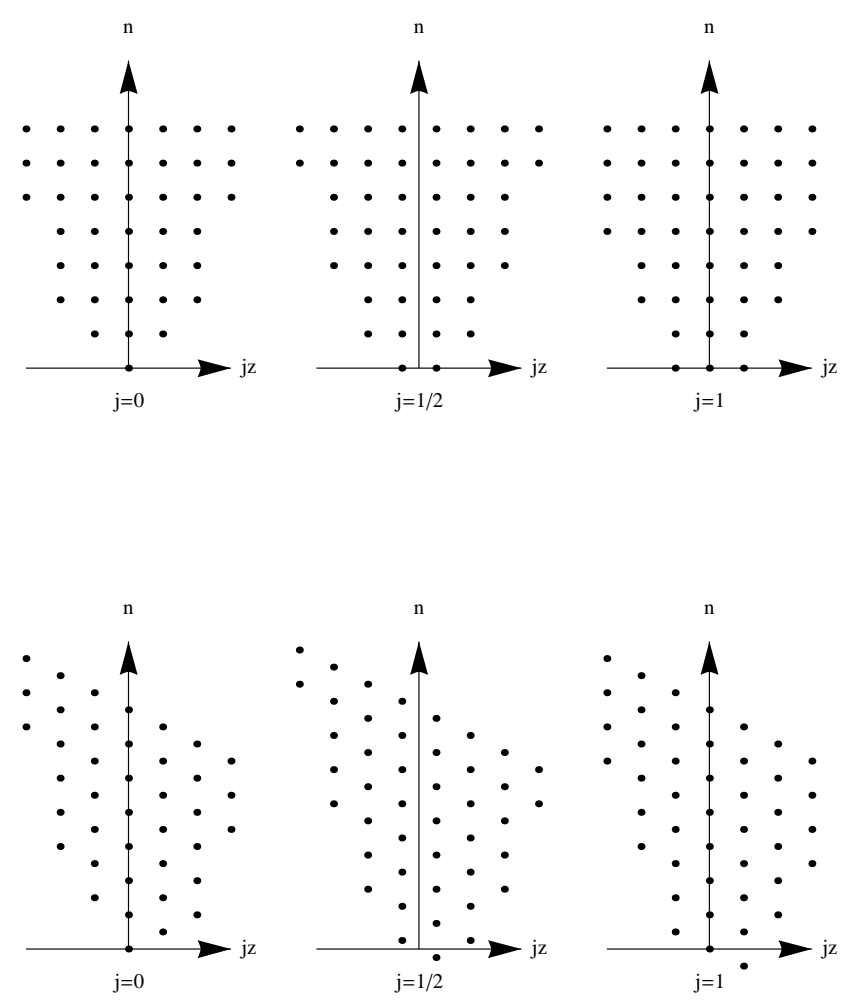

FIG. 1: Top panels: $S U(2)_{k}$ Kac-Moody conformal towers for $k=2$, for $j=0, \frac{1}{2}, 1$ from left to right. Bottom panels: tilted towers due to Zeeman term $-b j_{F}^{z}$ with $b=\frac{1}{2}$. Multiplicities are not shown.

is thus

$$
\Delta E=-\frac{1}{\pi} \sum_{\sigma \alpha} \delta_{\sigma \alpha} N_{\sigma \alpha}
$$

In terms of the particle numbers, the total charge is $Q=\sum_{\sigma}\left(N_{\sigma L}+N_{\sigma R}\right)$ while the magnetic quantum number of the flavor sector is $j_{F}^{z}=\frac{1}{2} \sum_{\sigma}\left(N_{\sigma L}-N_{\sigma R}\right)$. In the presence of $H_{p s}$, the spectrum thus follows as $\tilde{E}_{2 C K}=E_{2 C K}+\Delta E$, viz

$$
\tilde{E}_{2 C K}=\frac{Q^{2}}{8}+\frac{j(j+1)}{4}+\frac{j_{F}\left(j_{F}+1\right)}{4}-Q \frac{\delta_{L}+\delta_{R}}{2 \pi}-j_{F}^{z} \frac{\delta_{L}-\delta_{R}}{\pi},
$$

where $\delta_{\sigma \alpha} \equiv \delta_{\alpha}$ since the potential scattering $H_{p s}$ is independent of spin $\sigma$. Eq. (3) is equivalent to Eq. (4) of Ref. 1, with $a=2 \frac{\delta_{L}+\delta_{R}}{\pi}$ and $b=\frac{\delta_{L}-\delta_{R}}{\pi}$. Thus $H_{p s}$ simply biases the charge and flavor conformal towers. For a visualization of the effect on the $S U(2)_{2}$ flavor towers, see the lower panels of Fig. 1 The spectrum obtained using the trivial free Fermion gluing conditions ${ }^{3}$ describes free electrons but with the additional potential scattering. Importantly, switching on $H_{p s}$ and switching on the Kondo interactions commute, since there is a spin-charge-flavor separation in the model $\underline{\underline{3}}$ Employing instead the nontrivial gluing conditions of Table $\llbracket$ in Eq. (3), we thus obtain the 2CK critical spectrum including the influence of $H_{p s}$.

In Fig. 2, we examine the evolution of the energy levels at the critical point of the $2 \mathrm{CK}$ model as function of $\delta_{L}$ for $\delta_{R}=0$, plotting the lowest excitation energies $\delta E_{i}=\tilde{E}_{i}-\min \left\{\tilde{E}_{i}\right\}$. The fractions $0, \frac{1}{8}, \frac{1}{2}, \frac{5}{8}, 1 \ldots$ correspond to multiplets of the symmetry $U(1) \times S U(2)_{2} \times S U(2)_{2}$ of the regular 2 CK model. Those multiplets split due to the flavor field and charge field induced by finite $H_{p s}$, but eventually at $\delta_{L}=\pi / 2$ they recombine to give new multiplets characterized by the fractions $0, \frac{3}{8}, \frac{1}{2}, \frac{7}{8}, 1 \ldots$, and which correspond to that of the regular $2 \mathrm{IKM} \mathrm{s}^{\frac{5}{5}}$

To strengthen the connection between the two models, we now consider the effect of $H_{p s}$ on the critical CFT of the 2IKM itself. We exploit here the Bose-Ising representation $\underline{\underline{5}}$ of the 2IKM, consisting of a decomposition into $S U(2)_{1} \times S U(2)_{1} \times S U(2)_{2} \times Z_{2}$ left/right charge (isospin), total spin and $Z_{2}$ Ising symmetry sectors. The $z$-component of $\alpha=L$ or $R$ isospin is essentially the total charge of channel $\alpha$. Specifically, its magnetic quantum number is given 


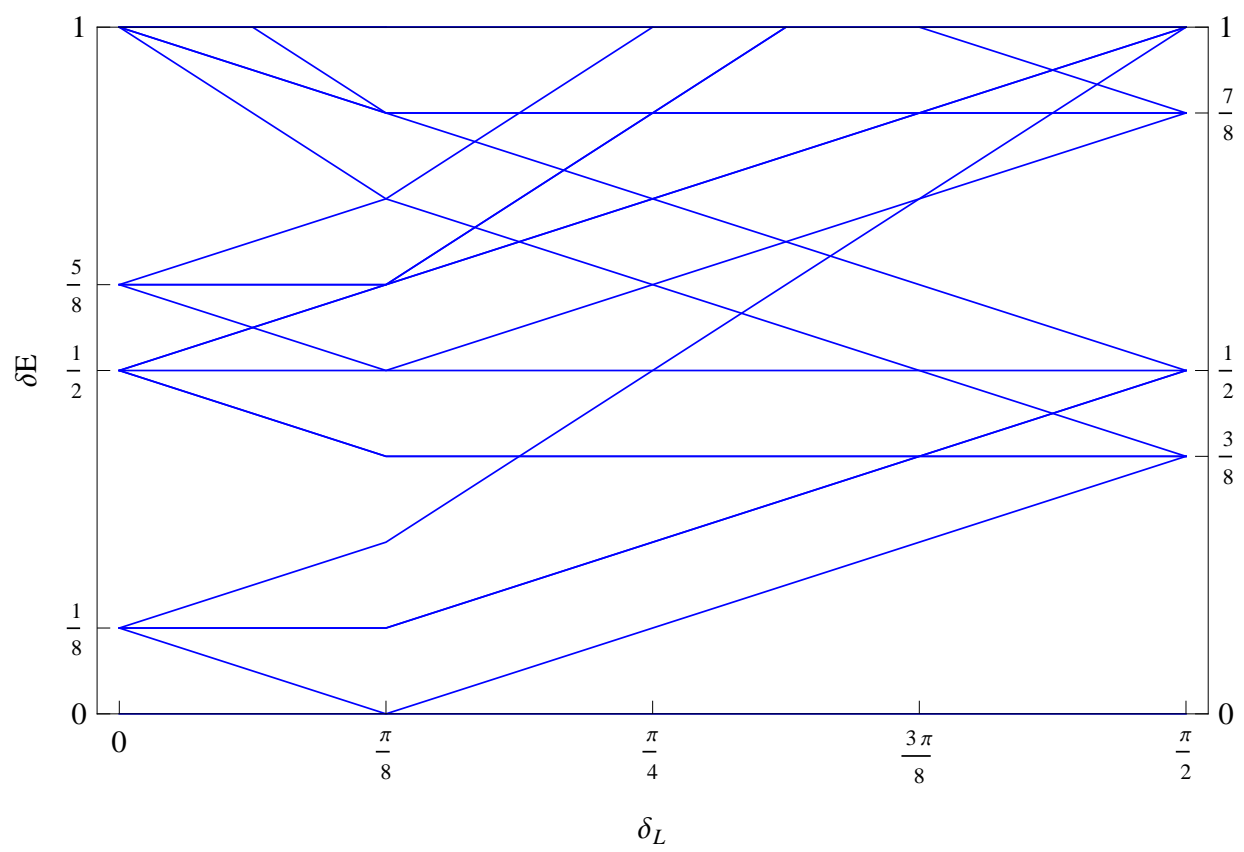

FIG. 2: Excitation energies $\delta E$ according to Eq. (3) as a function of $\delta_{L}$ for $\delta_{R}=0$, connecting the regular 2CK spectrum $\left(\delta_{L}=0\right)$ and the $2 \mathrm{IKM}$ spectrum $\left(\delta_{L}=\pi / 2\right)$. Note that at $\delta_{L}=\pi / 8$ the ground state changes from the $\operatorname{doublet}\left(Q, j, j_{F}\right)=(0,1 / 2,0)$ to the $j_{F}^{z}=1 / 2, Q=1$ component of the quintet $( \pm 1,0,1 / 2)$.

simply by $i_{\alpha}^{z}=\frac{1}{2} \sum_{\sigma} N_{\sigma \alpha}$ in terms of the particle numbers. Using Eq. (2), it follows that $\Delta E=-\frac{2}{\pi} \sum_{\alpha} \delta_{\alpha} i_{\alpha}^{z}$. The energy of the lowest states of each conformal tower in the $2 \mathrm{IKM}$ with the additional potential scattering term $H_{p s}$ is thus $\tilde{E}_{2 I K}=E_{2 I K}+\Delta E$, where $E_{2 I K}$ is given in Ref. $\underline{5}$. Our final result is

$$
\tilde{E}_{2 I K}=\frac{i_{L}\left(i_{L}+2\right)}{3}+\frac{i_{R}\left(i_{R}+2\right)}{3}+\frac{j(j+1)}{4}+x_{\text {Ising }}-2 \frac{\delta_{L}}{\pi} i_{L}^{z}-2 \frac{\delta_{R}}{\pi} i_{R}^{z},
$$

where, $i_{\alpha}=0, \frac{1}{2}$ are the isospin quantum numbers, $j=0, \frac{1}{2}, 1$ is the total spin quantum number and $x_{I s i n g}=0, \frac{1}{16}, \frac{1}{2}$ (corresponding to the unity operator, spin field $\sigma$, and fermion field $\epsilon$ of the $Z_{2}$ Ising model). The nontrivial gluing condition of the $S U(2)_{1} \times S U(2)_{1} \times S U(2)_{2} \times Z_{2}$ sectors is given in Table. III of Ref. 5 , and gives the finite size spectrum of the $2 \mathrm{IKM}$ in the presence of potential scattering.

Using Eqs. (3) and (4) with their corresponding gluing conditions, we find

$$
\mathcal{H}_{C P}^{2 I K}\left(\delta_{L}, \delta_{R}\right)=\mathcal{H}_{C P}^{2 C K}\left(\delta_{L}+\pi / 2, \delta_{R}\right),
$$

where $\mathcal{H}_{C P} \equiv\left\{\delta E_{i}\right\}$ at the critical point of either model, parametrized in terms of the free Fermion phase shifts $\delta_{L}$ and $\delta_{R}$. The finite size spectrum at the critical point of one model with potential scatterings $V_{L}$ and $V_{R}$ is identical to that of the other model with different $\tilde{V}_{L}$ and $\tilde{V}_{R}$ when the phase shifts satisfy Eq. (5). Thus, the critical FPs are equivalent in the sense that they lie on the same marginal manifold parametrized by $H_{p s}$.

\section{A. Alternative formulation in terms of Majorana Fermions}

Having drawn the connection between the non-abelian CFT formulation of the 2CK and 2IKM, we discuss now the same relation within the framework of abelian bosonization.

The steps applied first by Emery and Kivelson ${ }^{6}$ as an alternative solution to the $2 \mathrm{CK}$ model, and later by Gan ${ }^{7}$ in the 2IKM context, are as follows. (i) Bosonize separately each chiral Fermionic species (with the impurities at the 'boundary' located at $x=0), \psi_{\sigma \alpha}(x) \sim e^{-i \phi_{\sigma \alpha}(x)}$. (ii) Define linear combinations of the bosonic fields $\phi_{\sigma \alpha}(x)$, $\left\{\phi_{c}, \phi_{s}, \phi_{f}, \phi_{X}\right\}=\frac{1}{2} \sum_{\sigma, \alpha=1}^{2} \phi_{\sigma \alpha}\left\{1,(-1)^{\sigma+1},(-1)^{\alpha+1},(-1)^{\sigma+\alpha}\right\}$, corresponding to charge, spin, flavor and spin-flavor bosons $(A=c, s, f, X)$. (iii) Define four new Fermionic species by $\psi_{A}(x) \sim e^{-i \phi_{A}(x)}$, whose real $\left(\chi_{1}^{A}=\frac{\psi_{A}^{\dagger}+\psi_{A}}{\sqrt{2}}\right)$ and 
imaginary $\left(\chi_{2}^{A}=\frac{\psi_{A}^{\dagger}-\psi_{A}}{\sqrt{2} i}\right)$ parts give eight Majorana Fermions (MFs) $\chi_{j}^{A}(x)$, (with $j=1,2$ and $\left.A=c, s, f, X\right)$, which may be regarded as components of the vector $\vec{\chi}(x)$. The Hamiltonian for the free theory with $J_{L}=J_{R}=0$ and $H_{p s}=0$ is then $H=\frac{i}{2} \int_{-\infty}^{\infty} d x \vec{\chi}(x) \cdot \partial_{x} \vec{\chi}(x)$, with the trivial boundary condition $(\mathrm{BC}) \vec{\chi}\left(0^{+}\right)=\vec{\chi}\left(0^{-}\right)$. The $\mathrm{BC}$ relates the field before scattering at $x=0^{+}$and after scattering at $x=0^{-}$(using a left moving convention). The remarkable fact is that in both $2 \mathrm{CK}$ and $2 \mathrm{IKM}$, the critical FP Hamiltonian is the same as that of the free theory, but with a modified BC that is also simple in terms of the MFs. For $2 \mathrm{CK}$ it is simply $\underline{\underline{3}}$

$$
\vec{\chi}_{s}\left(0^{+}\right)=-\vec{\chi}_{s}\left(0^{-}\right) \quad \text { and } \quad \chi_{j}^{A}\left(0^{+}\right)=\chi_{j}^{A}\left(0^{-}\right) \text {for }(A, j) \neq(s, 1),(s, 2),(X, 1),
$$

with $\vec{\chi}_{s}=\left(\chi_{1}^{s}, \chi_{2}^{s}, \chi_{1}^{X}\right)$, such that the modified BC affects only the spin sector of the 2CK model. For $2 \mathrm{IKM}, \underline{\underline{8}}$

$$
\chi_{2}^{X}\left(0^{+}\right)=-\chi_{2}^{X}\left(0^{-}\right) \quad \text { and } \quad \chi_{j}^{A}\left(0^{+}\right)=\chi_{j}^{A}\left(0^{-}\right) \text {for }(A, j) \neq(X, 2),
$$

where the modified $\mathrm{BC}$ here affects only the Ising sector of the 2IKM.

An odd number of MFs suffer the modified BC in both models. This is a hallmark of NFL behavior, as it implies $\underline{8}^{8}$ a vanishing amplitude for scattering of an electron into an electron. By contrast, the BCs for Fermi liquids always involve an even number of MFs, corresponding thereby to linear BCs for regular Fermions.

Besides their common NFL character, the different BCs for the critical points of the 2CK and 2IKM do not immediately suggest any connection between the models. However, we now show that adding potential scattering in one channel of the $2 \mathrm{CK}$ model (Eq. (2) of Ref. 1, with $V_{L} \rightarrow \infty$ and $V_{R} \rightarrow 0$ in the added term $H_{p s}$ ) causes a change in the critical $2 \mathrm{CK} \mathrm{BC}$ which makes it equivalent to that of the 2IKM.

As above, the addition of infinite potential scattering in the left channel (which results in the desired $\delta_{L}=\pi / 2$ phase shift) is equivalent to the addition of infinite uniform potential scattering $\frac{1}{2} \psi_{L}^{\dagger} \psi_{L}+\frac{1}{2} \psi_{R}^{\dagger} \psi_{R}=i \chi_{2}^{c} \chi_{1}^{c}$ which affects only the charge sector, and infinite staggered potential scattering $\frac{1}{2} \psi_{L}^{\dagger} \psi_{L}-\frac{1}{2} \psi_{R}^{\dagger} \psi_{R}=i \chi_{2}^{f} \chi_{1}^{f}$, affecting only the flavor sector. Since both charge and flavor sectors are unaffected by the Kondo interaction (which acts purely in the spin sector), the resulting behavior can be understood from the response of free Fermions to potential scattering in the simpler situation $J_{L}=J_{R}=0$. For $V_{L} \rightarrow \infty$ and $V_{R} \rightarrow 0$, modified BCs are conferred to the charge and flavor

MFs: $\chi_{1,2}^{c, f}\left(0^{+}\right)=-\chi_{1,2}^{c, f}\left(0^{-}\right)$. Together with the BC from Eq. [6] we now obtain the BC for the critical $2 \mathrm{CK}$ model in the presence of the potential scattering, viz

$$
\chi_{2}^{X}\left(0^{+}\right)=\chi_{2}^{X}\left(0^{-}\right) \quad \text { and } \quad \chi_{j}^{A}\left(0^{+}\right)=-\chi_{j}^{A}\left(0^{-}\right) \text {for }(A, j) \neq(X, 2) .
$$

This BC is the same as that of the 2IKM, Eq. (7), except for an additional minus sign afflicting the BC for all MFs. However, the BCs corresponding to Eqs. (7) and (8) are in fact equivalent in the 2CK model, as now shown.

An 'overall' minus sign in the BC for the MFs can be interpreted as a phase shift felt by each of the charge, spin, flavor and spin-flavor Fermions, defined in terms of the scattering process $\psi_{A}\left(0^{-}\right)=\exp \left(2 i \delta_{A}\right) \psi_{A}\left(0^{+}\right)$, and with $\delta_{A}=\pi / 2$ for $A=c, s, f, X$. Since the number operators for $\psi_{A}$ Fermions are related 9 to the number operators for the regular $\psi_{\sigma \alpha}$ Fermions via $\left\{N_{c}, N_{s}, N_{f}, N_{X}\right\}=\frac{1}{2} \sum_{\sigma \alpha} N_{\sigma \alpha}\left\{1,(-1)^{\sigma+1},(-1)^{\alpha+1},(-1)^{\sigma+\alpha}\right\}$, it follows that $\sum_{A} N_{A}=2 N_{\uparrow L}$. In terms of the particle numbers $N_{A}$, the correction to the total energy due to additional phase shifts is $\Delta E=-\frac{1}{\pi} \sum_{A} \delta_{A} N_{A}=-N_{\uparrow L}$. Comparing to Eq. 2, we then find that $\delta_{\uparrow L}=\pi$ (and $\delta_{\sigma \alpha}=0$ for $\left.\sigma \alpha \neq \uparrow L\right)$. Thus the additional global minus sign in the BCs for the MFs equates to $\psi_{\uparrow L}\left(0^{-}\right)=\exp \left(2 i \delta_{\uparrow L}\right) \psi_{\uparrow L}\left(0^{+}\right) \equiv \psi_{\uparrow L}\left(0^{+}\right)$ for the original Fermions, and so has no effect on the finite size spectrum — or on the lowest-energy physics in general.

\section{CORRECTION TO THE GREEN FUNCTION NEAR THE 2IKM CRITICAL POINT}

In this appendix we consider corrections to the critical FP Hamiltonian of the 2IKM, and from them construct corrections to the Green function. We show that the nature of these corrections (which show up in physical quantities such as conductance) is characteristic of the NFL behavior, and depend distinctively on parity breaking in the model.

\section{A. Fixed point Hamiltonian of the 2IKM}

First we construct the list of operators allowed by symmetry in the generic asymmetric 2IKM (see Eq. (1) of Ref. 1), including the dependence on small $J_{L}-J_{R}$ and on small potential scattering amplitudes $V_{L}$ and $V_{R}$. We rely on the

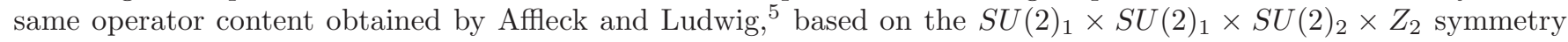
decomposition. The critical FP of the symmetric 2IKM without potential scattering has only one irrelevant operator of dimension 3/2, denoted $\epsilon^{\prime}$, and is given by Eq. (5.1) of Ref. 5. In the present context where parity and particle-hole 


\begin{tabular}{|rrrrr|}
\hline$i_{L}$ & $i_{R}$ & $j$ & Ising & $x$ \\
\hline 0 & 0 & 0 & 1 & 0 \\
$\frac{1}{2}$ & 0 & $\frac{1}{2}$ & $\sigma$ & $\frac{1}{2}$ \\
0 & $\frac{1}{2}$ & $\frac{1}{2}$ & $\sigma$ & $\frac{1}{2}$ \\
0 & 0 & 1 & 1 & $\frac{1}{2}$ \\
0 & 0 & 0 & $\epsilon$ & $\frac{1}{2}$ \\
$\frac{1}{2}$ & $\frac{1}{2}$ & 0 & 1 & $\frac{1}{2}$ \\
0 & 0 & 1 & $\epsilon$ & 1 \\
$\frac{1}{2}$ & $\frac{1}{2}$ & 1 & 1 & 1 \\
$\frac{1}{2}$ & $\frac{1}{2}$ & 0 & $\epsilon$ & 1 \\
$\frac{1}{2}$ & $\frac{1}{2}$ & 1 & $\epsilon$ & $\frac{3}{2}$ \\
\hline
\end{tabular}

TABLE II: Operator content of the 2IKM critical FP.

symmetries are in general broken, additional operators are allowed, and in fact dominate the approach to the NFL FP.

Each operator that can occur at an unstable FP is either a primary operator or a descendant of it. $\frac{5}{\underline{\underline{T}}}$ The primary operators are labeled with the same quantum numbers $\left(i_{1}, i_{2}, j\right.$, Ising) used to label states in Eq. (4). The particular combinations of $S U(2)_{1} \times S U(2)_{1} \times S U(2)_{2} \times Z_{2}$ left/right isospin, total spin and Ising operators, together with their scaling dimension $x$, was obtained using the double fusion method in Ref. 5 . For clarity and completeness we reproduce that list here in Table II

In general, the presence of a given symmetry implies that the only allowed operators are those which are singlets of that symmetry. In particular, the full $S U(2)_{1} \times S U(2)_{1} \times S U(2)_{2} \times Z_{2}$ symmetry of the standard 2 IKM with $H_{p s}=0$ gives rise to the leading singlet operator $\left(i_{1}, i_{2}, j, \mathrm{Ising}\right)=(0,0,0, \epsilon)$. This relevant operator is associated ${ }^{5}$ with the perturbation $\left(K-K_{c}\right)$, which destabilizes the 2 IKM critical point. At the critical point $K=K_{c}$, one leading irrelevant operator ${ }^{5}$ is the descendant of $(0,0,0, \epsilon)$, denoted $\mathcal{O}_{1}=\epsilon^{\prime} \approx \partial_{\tau} \epsilon$. Since $\epsilon$ has scaling dimension $1 / 2$, its first descendant has scaling dimension $3 / 2$. But singlets can also be obtained as descendants of primary $S U(2)$ fields with integer spin. Indeed, the other singlet operator with dimension $3 / 2$ is in this case obtained by acting on the $\vec{\phi}=(0,0,1,1)$ primary vector field of the $j=1$ spin sector with an operator that creates spin excitations $\underline{\underline{5}}$ Thus, one must consider also a second irrelevant operator $\mathcal{O}_{2}=\vec{J}_{-1} \cdot \vec{\phi}$ (where $\vec{J}_{-1}$ is the lowest Fourier mode of the spin current creating such excitations).

Since $\vec{J}_{-1} \cdot \vec{\phi}$ is also the leading irrelevant operator in the $2 \mathrm{CK}$ model, 10 one might expect the physical behavior stemming from it to be common to both $2 \mathrm{CK}$ and $2 \mathrm{IKM}$. However, the crucial difference between the models is that $\vec{J}_{-1} \cdot \vec{\phi}$ has odd parity in the $2 \mathrm{IKM}$ but even parity in the $2 \mathrm{CK}$ model; as now shown.

Parity symmetry (corresponding to left/right reflection in space) is defined by invariance under the permutations $\vec{S}_{L} \leftrightarrow \vec{S}_{R}$ and $\psi_{L} \leftrightarrow \psi_{R}$. One rather subtle consequence ${ }^{\underline{5}}$ of parity symmetry in the 2 IKM is that $\vec{\phi} \rightarrow-\vec{\phi}$. This can be seen from the parity-odd operator $\psi_{L}^{\dagger} \vec{\sigma} \psi_{L}-\psi_{R}^{\dagger} \vec{\sigma} \psi_{R}=\vec{\phi} \epsilon$ (and noting that $\epsilon$ is the operator corresponding to $\left(K-K_{c}\right)$, and is as such unaffected by the reflection, $\left.\epsilon \leftrightarrow \epsilon\right)$. Thus $\vec{J}_{-1} \cdot \vec{\phi}$ is also parity-odd in the 2 IKM. But in the 2CK model, $\psi_{L}^{\dagger} \vec{\sigma} \psi_{L}-\psi_{R}^{\dagger} \vec{\sigma} \psi_{R}=\vec{\phi} \phi_{F}^{z}$. Here, $\vec{\phi}_{F}$ is the $j_{F}=1$ primary field of the flavor sector, and $\phi_{F}^{z}$ is its $z$-component. Since parity corresponds to a $\pi$ rotation around the $x$ flavor direction in the $2 \mathrm{CK}$ model, the flavor current $\vec{J}_{F}=\sum_{\sigma} \psi_{\sigma}^{\dagger} \frac{\vec{\tau}}{2} \psi_{\sigma}$ transforms as $J_{F}^{x} \rightarrow J_{F}^{x}, J_{F}^{y, z} \rightarrow-J_{F}^{y, z}$; and similarly $\phi_{F}^{x} \rightarrow \phi_{F}^{x}, \phi_{F}^{y, z} \rightarrow-\phi_{F}^{y, z}$. Thus $\vec{J}_{-1} \cdot \vec{\phi}$ is parity even in the $2 \mathrm{CK}$ model. Of course, this is the expected result here since $\vec{J}_{-1}$ and $\vec{\phi}$ are both spin operators, but the parity transformation affects only the flavor sector of the $2 \mathrm{CK}$ model.

Finally, we consider the additional operators appearing when isospin $S U(2)_{1} \times S U(2)_{1}$ symmetry is broken (but spin $S U(2)$ symmetry is maintained), as occurs when potential scattering from $H_{p s}$ is included. Specifically, $H_{p s}=$ $2 \sum_{\alpha} V_{\alpha} I_{\alpha}^{z}(x=0)$, where the $z$-component of the channel- $\alpha$ isospin current is given by $I_{\alpha}^{z}(x)=\frac{1}{2} \sum_{\sigma} \psi_{\sigma \alpha}^{\dagger}(x) \psi_{\sigma \alpha}(x)$. Since $V_{\alpha}$ does not couple to fields with half-integer isospin, and the primary operators have total spin $j=0$, then it follows that $V_{\alpha}$ couples only to dimension $3 / 2$ descendants of the $(0,0,0, \epsilon)$ field. Thus, two further dimension $3 / 2$ operators $\mathcal{O}_{3,4}=\left(I_{L, R}^{z}\right)_{-1} \epsilon$ are obtained (where $\left(\vec{I}_{\alpha}\right)_{-1}$ is the isospin analogue of the spin operator $\vec{J}_{-1}$ discussed above). No other dimension $3 / 2$ operators are consistent with the symmetry. 
The corrections to the critical FP Hamiltonian in the presence of $H_{p s}$ thus comprise four operators,

$$
\delta H=\frac{1}{\sqrt{T_{c}}} \sum_{i=1}^{4} c_{i} \mathcal{O}_{i} .
$$

As shown in Ref. $\underline{5}$, the coefficient $c_{1} \sim 1$. By contrast, the odd transformation property of $\mathcal{O}_{2}$ under parity implies

$$
c_{2} \sim\left(\frac{J_{L}-J_{R}}{J_{L}+J_{R}}\right)
$$

for small small $\left(J_{L}-J_{R}\right)$. Finally $c_{3,4} \propto V_{L, R}$ for small $V_{L, R}$.

In the conformal limit (which relies upon linear dispersion and infinite conduction bandwidth), the Bose-Ising representation of the $2 \mathrm{IKM}$ reads $H_{2 I K}=H_{0}+\vec{J}(0) \cdot\left(J_{L} \vec{S}_{L}+J_{R} \vec{S}_{R}\right)+\vec{\phi}(0) \epsilon \cdot\left(J_{L} \vec{S}_{L}-J_{R} \vec{S}_{R}\right)+2 \sum_{\alpha} V_{\alpha} I_{\alpha}^{z}(0)$, showing that there is perfect separation of isospin and Ising sectors (although there is a coupling between Ising and spin via the term $\vec{\phi}(0) \epsilon)$. Since $\mathcal{O}_{3,4}$ involve coupling between isospin and Ising operators $\left(I_{L, R}^{z}\right)_{-1}$ and $\epsilon$, in this idealized limit one thus obtains $c_{3,4}=0$ identically. But in generic models, one naturally expects $c_{3,4} \ll 1$ to be finite but small. For this reason we ignore the operators $\mathcal{O}_{3,4}$ in the present work.

However, we do note that the $\mathcal{O}_{3,4}$ operators do play a significant role in variants of the standard 2IKM which contain explicit coupling between isospin and Ising or spin sectors. The two-impurity Anderson model is a pertinent example: the low-energy effective mode $\underline{1}^{5.11}$ is a $2 \mathrm{IKM}$ but with additional terms such as $\psi^{\dagger}\left(\vec{\sigma} \tau^{x}\right) \psi \cdot\left(\vec{S}_{L}+\vec{S}_{R}\right)$. These terms mix the spin and isospin sectors, leading thereby to indirect coupling between $\left(I_{L, R}^{z}\right)_{-1}$ and $\epsilon$ operators, and hence in such situations $c_{3,4}$ are not expected to be small.

\section{B. Corrections to the Green function}

Corrections to the $\mathrm{t}$ matrix and conductance in the vicinity of the $2 \mathrm{IKM}$ critical point are directly related to corrections to the single particle Green function $\underline{10}$ Ignoring the operators $\mathcal{O}_{3,4}$ as above, the leading correction to the Green function to first order in Eq. (9) is $\delta G\left(z_{1}, z_{2}\right)=\delta_{1} G\left(z_{1}, z_{2}\right)+\delta_{2} G\left(z_{1}, z_{2}\right)$, where

$$
\begin{aligned}
& \delta_{1} G\left(z_{1}, z_{2}\right)=\frac{c_{1}}{\sqrt{T_{c}}} \int_{0}^{\beta} d \tau\left\langle\psi\left(z_{1}\right) \epsilon^{\prime}(0, \tau) \psi^{\dagger}\left(z_{2}\right)\right\rangle, \\
& \delta_{2} G\left(z_{1}, z_{2}\right)=\frac{c_{2}}{\sqrt{T_{c}}} \int_{0}^{\beta} d \tau\left\langle\psi\left(z_{1}\right) \vec{J}_{-1} \cdot \vec{\phi}(0, \tau) \psi^{\dagger}\left(z_{2}\right)\right\rangle,
\end{aligned}
$$

and $\beta=1 / T$ is inverse temperature. Here we suppress the spin and channel indices, and use $z_{1}=\tau_{1}+i r_{1}, \bar{z}_{2}=\tau_{2}+i r_{2}$. Assuming $r_{1}>0$ and $r_{2}<0$, the propagator is sensitive to scattering from the impurities at the boundary located at $r=0$. The calculation of $\delta_{2} G\left(z_{1}, z_{2}\right)$ was performed by Affleck and Ludwig,$\frac{10}{w}$ who exploited the fact that the three-point function in $\delta_{2} G\left(z_{1}, z_{2}\right)$ is fully determined by conformal invariance (up to an overall constant) since $\vec{J}_{-1} \cdot \vec{\phi}$ is a Virasoro primary. The electron and Ising fields, $\psi$ and $\epsilon$, are chiral fields with scaling dimension $1 / 2$, and thus $\frac{10}{2}$

$$
\begin{array}{r}
\delta_{2} G\left(z_{1}, z_{2}\right) \propto \frac{c_{2}}{\sqrt{T_{c}}}\left(\frac{\pi}{\beta}\right)^{5 / 2} \times \\
\int_{0}^{\beta} d \tau \frac{\left[\sin \frac{\pi}{\beta}\left(z_{1}-z_{2}\right)\right]^{1 / 2}}{\left[\sin \left(\frac{\pi}{\beta}\left(\tau-z_{1}\right)\right) \sin \left(\frac{\pi}{\beta}\left(\tau-z_{2}\right)\right)\right]^{3 / 2}} .
\end{array}
$$

The $\tau$ integral can be expressed in terms of hypergeometric functions, which yield asymptotically the famous $\sqrt{\omega}$ energy-dependence of the related t matrix $\underline{10}$

The integrand in $\delta_{1} G\left(z_{1}, z_{2}\right)$ is similarly a three point function, but $\epsilon^{\prime} \approx \partial_{\tau} \epsilon$ is not a Virasoro primary field. However, $\epsilon$ itself is Virasoro primary. Pulling the derivative out of the correlation function, one obtains

$$
\begin{array}{r}
\delta_{1} G\left(z_{1}, z_{2}\right)=\frac{c_{1}}{\sqrt{T_{c}}} \int_{0}^{\beta} d \tau \partial_{\tau}\left\langle\psi\left(z_{1}\right) \epsilon(0, \tau) \psi^{\dagger}\left(z_{2}\right)\right\rangle \\
\frac{c_{1}}{\sqrt{T_{c}}}\left[\sin \frac{\pi}{\beta}\left(z_{1}-z_{2}\right)\right]^{-1 / 2}\left(\frac{\pi}{\beta}\right)^{3 / 2} \int_{0}^{\beta} d \tau \partial_{\tau} \frac{1}{\left[\sin \left(\frac{\pi}{\beta}\left(\tau-z_{1}\right)\right) \sin \left(\frac{\pi}{\beta}\left(\tau-z_{2}\right)\right)\right]^{1 / 2}}=0 .
\end{array}
$$




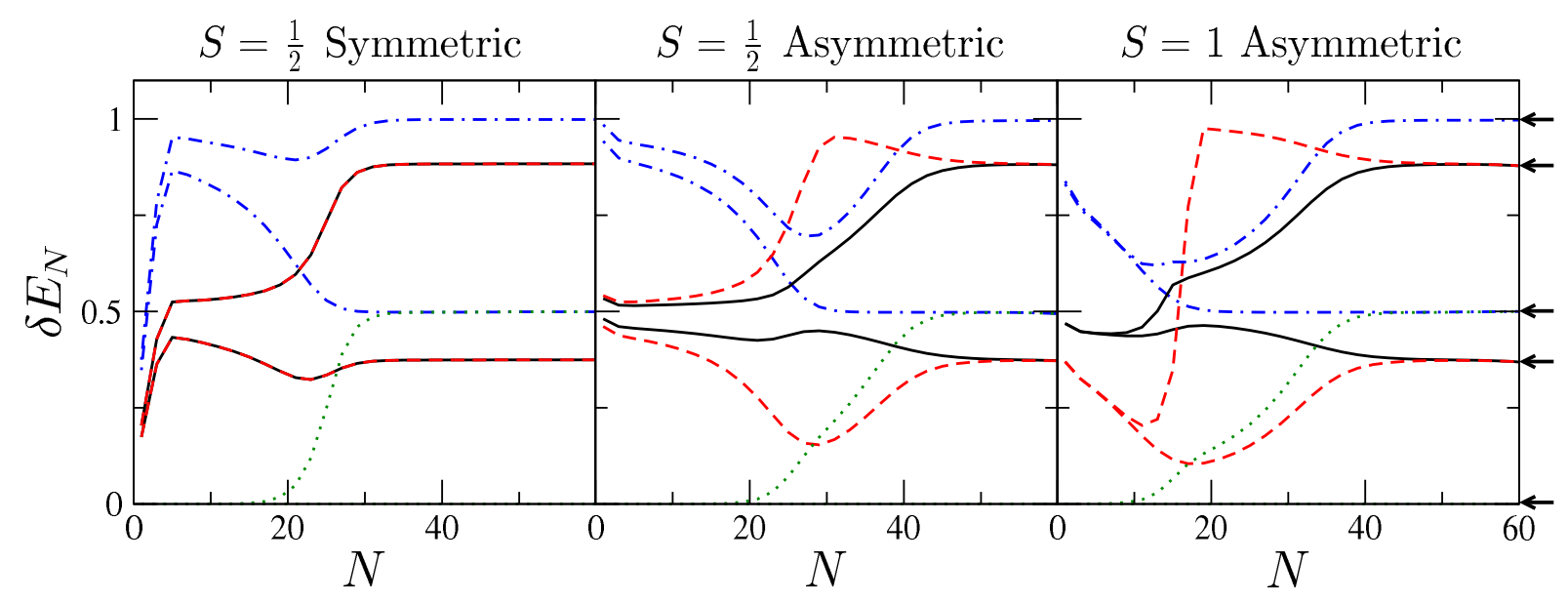

FIG. 3: NRG energy levels $\delta E_{N}$ for odd iteration number, N. The lowest 2 levels are shown in each left/right charge and total spin subspace with quantum numbers $\left(Q_{L}, Q_{R}, S_{\text {tot }}^{z}\right)=(0,0,0)$ [dotted lines], $\left(0,1, \frac{1}{2}\right)$ [solid lines], $\left(1,0, \frac{1}{2}\right)$ [dashed lines] and $(1,1,0)$ [dot-dashed lines]. Left panel: symmetric spin- $\frac{1}{2} 2 \mathrm{IKM}$ with $\rho J_{L}=\rho J_{R}=0.075$. Middle panel: asymmetric spin- $\frac{1}{2}$ 2IKM with $\rho J_{L}=0.075$ and $\rho J_{R}=0.05$. Right panel: asymmetric spin-1 $2 \mathrm{IKM}$ with $\rho J_{L}=0.15$ and $\rho J_{R}=0.05$. Interimpurity coupling $K \simeq K_{c}$ tuned to its critical value in each case, and $H_{p s}=0$.

The integrand has no singularities as long as $z_{1}$ and $z_{2}$ are away from the boundary, and no branch cuts associated with the square-root function are intersected. Periodicity in $\beta$ then implies that the integral vanishes, and so the contribution to the Green function from $\delta_{1} G\left(z_{1}, z_{2}\right)$ also vanishes. Thus, the anomalous square-root correction to the Green function must come from $\delta_{2} G\left(z_{1}, z_{2}\right)$ alone. In particular, our conclusion is that the coefficient of the square-root energy-dependence of the t matrix and hence conductance is proportional to $c_{2}$, which vanishes in the symmetric $2 \mathrm{IKM}$, obtained as $J_{L} \rightarrow J_{R}$.

Higher-order corrections to the Green function can be calculated in a similar fashion. In particular, the correction coming from the next order in perturbation theory involves integrals such as $\int_{0}^{\beta} d \tau \int_{0}^{\beta} d \tau^{\prime}\left\langle\psi\left(z_{1}\right) \mathcal{O}_{i}(0, \tau) \mathcal{O}_{i}\left(0, \tau^{\prime}\right) \psi^{\dagger}\left(z_{2}\right)\right\rangle$. Since such integrals contains singularities when $\tau \rightarrow \tau^{\prime}$, the correction does not in general vanish, even when $\partial_{\tau}$ and $\partial_{\tau^{\prime}}$ are pulled outside the correlator in the case of $\mathcal{O}_{1}=\epsilon^{\prime}$. Such calculations are notoriously involved; and here necessitate the use of an ultraviolet cutoff $\mathcal{O}\left(T_{c}\right)$ to avoid unphysical divergences. The leading correction is however expected to yield a linear energy dependence (albeit up to possible $\log$ corrections), in agreement with our NRG calculations, see Fig. 2 of Ref. 1 .

\section{2IKM CRITICAL PHYSICS WITHIN THE NUMERICAL RENORMALIZATION GROUP}

In this appendix we consider the role of parity breaking on the RG flow, finite size spectrum and thermodynamics of the 2IKM. Such information can be extracted from NRG calculations, $\stackrel{12}{=}$ as now briefly reviewed.

Since its first application to the single-impurity single-channel Kondo model,, 2 Wilson's NRG technique has been used successfully to study a wide range of quantum impurity problems (for a comprehensive recent review, see Ref. 13). More recently, the increase in computing resources has permitted the detailed analysis of two-channel models involving several impurities, such as the 2IKM considered in the present work.

The key element of the NRG technique $\underline{\underline{12}, 13}$ is a logarithmic discretization of the free conduction electron Hamiltonian, $H_{0}$. The continuum of states in each conduction band is divided into intervals with discretization points $x_{n}= \pm D \Lambda^{-n}$ (here $n=0,1,2, \ldots$ and $2 D$ is the bandwidth), and whose width thus decreases exponentially as the Fermi level is approached. A single state (the symmetric linear combination) is then retained in each interval, such that low-energies are exponentially-well resolved. Canonical transformation by tridiagonalization yields the Wilson chain representation, $\stackrel{12,13}{=}$ where each conduction channel corresponds to a semi-infinite chain terminated by the impurity sub-system. The discretized Hamiltonian is then diagonalized iteratively: starting from the impurity, Wilson chain orbitals are coupled on successively and the system diagonalized. To avoid exponential growth of the Hilbert space, high-energy states are discarded after each step. This truncation scheme correctly allows calculation of the lowest-energy eigenenergies $\left\{E_{N}\right\}$ of a finite Wilson chain of length $N$ because the coupling between the Wilson chain orbitals $N$ and $(N+1)$ scale as $\Lambda^{-N / 2}$. In consequence,,$\frac{12,13}{, 2}$ high-lying states at one iteration do not cross over and become low-lying states at a later iteration due to the energy scale separation inherent when $\Lambda>1$. 


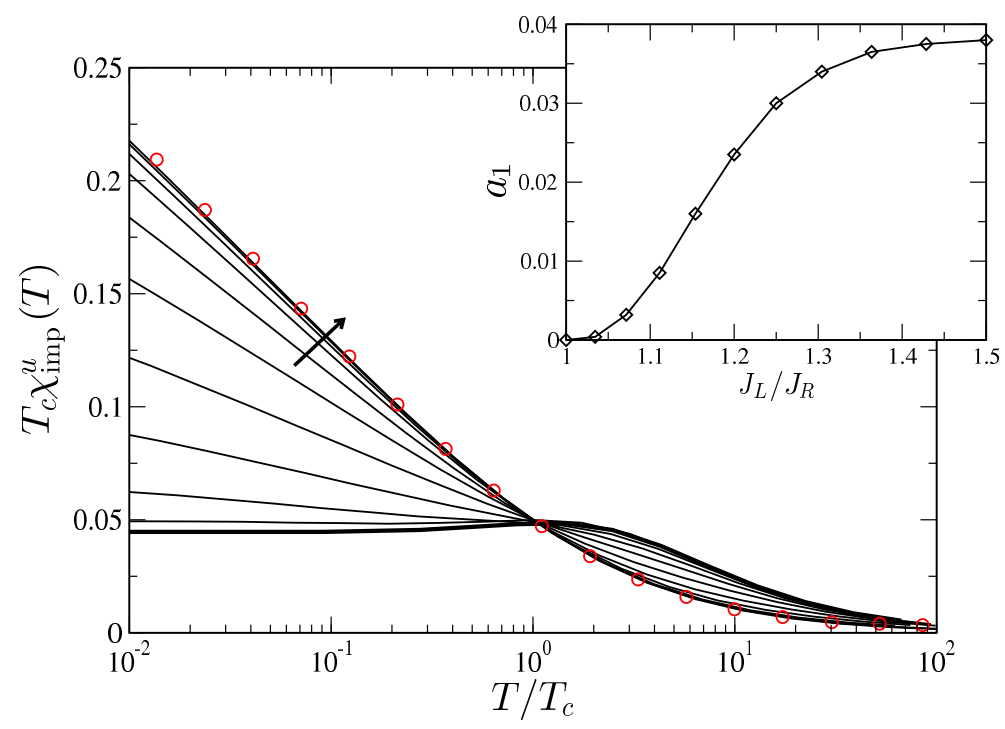

FIG. 4: Uniform magnetic susceptibility $T_{c} \chi_{\mathrm{imp}}^{u}(T)$ vs temperature $T / T_{c}$ for the $2 \mathrm{IKM}$ model. Shown for $\rho J_{L}=0.075 \geq \rho J_{R}$, varying $\rho J_{R}=0.075 \rightarrow 0.05$ in steps of 0.0025 , with $K=K_{c}$ retuned in each case (and $H_{p s}=0$ ), as in Fig. 2 of Ref. 1 . Thick solid line is the symmetric case $J_{L}=J_{R}$; asymmetry $J_{L} / J_{R} \geq 1$ increases in direction of the arrow. Circle points for a 2 CK model with $T_{K}^{2 C K} \equiv T_{c}$. Inset shows the variation of the slope $a_{1}$ as a function of $J_{L} / J_{R}$ (see Eq. 14). $a_{2} \approx 0.05$ is essentially independent of asymmetry. We have defined $T_{c}$ here through $T_{c} \chi_{\mathrm{imp}}^{u}\left(T_{c}\right)=0.05$.

$\delta E_{N}(i)=\Lambda^{N / 2}\left(E_{N}(i)-\min \left\{E_{N}\right\}\right)$ are rescaled many-particle energies (indexed $i$ and measured with respect to the ground state energy), and span roughly the same energy range, independent of $N$. The evolution of these levels with

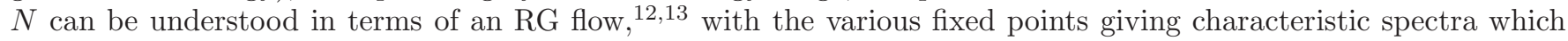
do not change upon further iteration. Indeed, the lowest energy levels at the stable fixed point (obtained as $N \rightarrow \infty$ ) reproduce accurately the finite size spectrum obtained by CFT $\underline{14}$

Thermodynamics can also be calculated from these NRG energy levels $\underline{12,13}$ The essential step here is the identification of a characteristic temperature $T_{N} \sim \Lambda^{-N / 2}$, at which thermodynamic quantities can be accurately calculated for a given finite iteration $N$. This temperature is chosen to be high enough that the splitting of the levels incurred at later iterations does not affect the thermodynamic calculation; but not too high that states above the truncation limit contribute significantly. Thus useful physical information can be extracted from each iteration, and so the full temperature-dependence of thermodynamic quantities can be built up.

\section{A. Effect of parity-braking on RG flow and finite size spectrum}

In Fig. 3 we show the evolution of low-lying NRG eigenenergies $\delta E_{N}$ with iteration number (Wilson chain length) $N$, for 2IKMs at criticality. Specifically, we compare the channel-symmetric and -asymmetric spin- $\frac{1}{2} 2 \mathrm{IKM}$ and the asymmetric spin-1 2IKM (with $H_{p s}=0$ in each case). The RG flow, as evidenced by the flow of these levels, is manifestly different for the three cases considered. In particular, levels with left/right charge and total spin quantum numbers $\left(Q_{L}, Q_{R}, S_{\mathrm{tot}}^{z}\right)=\left(0,1, \frac{1}{2}\right)$ and $\left(1,0, \frac{1}{2}\right)$ are of course degenerate in the symmetric 2IKM (left panel), since the Hamiltonian is invariant on swapping $L$ and $R$ labels. By contrast no such symmetry of the bare Hamiltonian is present in the channel-asymmetric spin- $\frac{1}{2}$ or spin-1 2IKMs plotted in the middle and right panels; and thus levels related by $L \leftrightarrow R$ permutation are not in general degenerate.

Importantly however, an emergent parity symmetry at the stable NFL fixed point is observed, with $\left(Q_{L}, Q_{R}, S_{\text {tot }}^{z}\right)$ and $\left(Q_{R}, Q_{L}, S_{\text {tot }}^{z}\right)$ levels becoming degenerate as $N \rightarrow \infty$.

Indeed, the set of NFL fixed point levels in each case is identical, demonstrating that the stable fixed point itself is identical, irrespective of bare model symmetries, and independent of spin- $S$. The fixed point levels are indicated by the arrows, and correspond to the fractions $0, \frac{3}{8}, \frac{1}{2}, \frac{7}{8}, 1, \ldots$, as obtained for the regular symmetric 2IKM by CFT $\stackrel{5}{\frac{5}{4}}$ 


\section{B. Effect of parity-braking on thermodynamics}

We turn now to thermodynamics, focusing on the 'impurity' contribution 12,13 to the uniform spin susceptibility, $\chi_{\mathrm{imp}}^{u}(T)=\left\langle\left(\hat{S}_{\mathrm{tot}}^{z}\right)^{2}\right\rangle_{\mathrm{imp}} / T$ (here $\hat{S}_{\text {tot }}^{z}$ refers to the spin of the entire system and $\langle\hat{\Omega}\rangle_{\mathrm{imp}}=\langle\hat{\Omega}\rangle-\langle\hat{\Omega}\rangle_{0}$, with $\langle\hat{\Omega}\rangle_{0}$ denoting a thermal average in the absence of the impurities).

In the $2 \mathrm{CK}$ model, the uniform susceptibility diverges logarithmically $\stackrel{15,16}{=}$ at low temperatures $T \ll T_{K}^{2 C K}$,

$$
T_{K}^{2 C K} \chi_{\mathrm{imp}}^{u}(T)=a_{1} \ln \left(T_{K}^{2 C K} / T\right)+a_{2} .
$$

One naturally expects the uniform susceptibility of the 2IKM to behave similarly in the channel-asymmetric limit (with $T_{K}^{2 C K} \equiv T_{c}$ ), since here there is a mapping to the $2 \mathrm{CK}$ model $\stackrel{2}{2}$ However, as pointed out in Ref. 5 , the uniform susceptibility is not singular in the regular symmetric 2IKM. Rather, it is the staggered susceptibility that is divergent in this case.

In Fig. 团we show how the uniform susceptibility $T_{c} \chi_{\text {imp }}^{u}(T)$ vs temperature $T / T_{c}$ evolves with increasing asymmetry for the spin- $\frac{1}{2}$ 2IKM. In the case of large channel asymmetry $\left(J_{L} / J_{R}=1.5\right)$, the behavior is indeed that of Eq. (14), with coefficients $a_{1}$ and $a_{2}$ essentially those of the regular 2CK model (see comparison to the pure 2CK case, circle points). But, in analogy to the vanishing square-root energy dependence of conductance at the symmetric point, we find that the coefficient $a_{1} \rightarrow 0$ as $J_{L} / J_{R} \rightarrow 1$ (see inset). Indeed, the leading contribution to the uniform susceptibility in this limit can be understood ${ }^{3}$ from second-order perturbation theory in the leading irrelevant operator $\mathcal{O}_{2}=\vec{J}_{-1} \cdot \vec{\phi}$. From Eq. (10) it then follows that $a_{1} \propto\left(J_{L}-J_{R}\right)^{2}$ for small $\left(J_{L}-J_{R}\right)$. Thus, there is a smooth crossover between the limiting cases, with divergent $2 \mathrm{CK}$ behavior arising in the asymmetric limit, but constant uniform susceptibility as $T \rightarrow 0$ emerging at the symmetric point, consistent with Ref. 5 .

1 A. K. Mitchell, E. Sela and D. E. Logan (unpublished).

2 G. Zaránd, C.-H. Chung, P. Simon, and M. Vojta, Phys. Rev. Lett. 97, 166802 (2006).

3 I. Affleck and A. W. W. Ludwig, Nucl. Phys. B 360, 641 (1990).

${ }^{4}$ I. Affleck, D. Gepner, H. J. Schulz and T. Ziman J. Phys. A: Math. Gen. 22511 (1989).

5 I. Affleck, A. W. W. Ludwig, and B. A. Jones, Phys. Rev. B 52, 9528 (1995).

${ }^{6}$ V. J. Emery and S. Kivelson, Phys. Rev. B 46, 10812 (1992).

7 J. Gan, Phys. Rev. Lett. 74, 2583 (1995).

8 J. M. Maldacena and A. W. W. Ludwig, Nucl. Phys. B 565, 506 (1997).

${ }^{9}$ G. Zaránd and J. von Delft, Phys. Rev. B 61, 6918 (2000).

${ }^{10}$ I. Affleck and A. W. W. Ludwig, Phys. Rev. B 48, 7297 (1993).

11 F. W. Jayatilaka, M. R. Galpin, and D. E. Logan, Phys. Rev. B 84, 115111 (2011).

12 K. G. Wilson, Rev. Mod. Phys. 47, 773 (1975).

13 R. Bulla, T. Costi, and T. Pruschke, Rev. Mod. Phys. 80, 395 (2008).

14 Strictly, the finite size spectrum is obtained on taking the $\Lambda \rightarrow 1$ limit 17 However, the lowest fixed point levels of the NRG recover the CFT spectrum to within a few percent for $\Lambda=3$, as employed in the present work.

15 N. Andrei and C. Destri, Phys. Rev. Lett. 52, 364 (1984).

16 A. M. Tsvelik, J. Phys. C 18, 159 (1985).

17 R. Bulla, A. C. Hewson, and G.-M. Zhang, Phys. Rev. B 56, 11721 (1997). 